Optics and Lasers in Engineering, Volume 137, February 2021, Article number 106342

DOI:10.1016/j.optlaseng.2020.106342

\title{
Three-dimensional interferometric stage encoder using a single access port
}

\author{
Kieran B. Wiseman, Thomas Kissinger*, Ralph P. Tatam \\ Centre for Engineering Photonics, Cranfield University, Cranfield, MK43 0AL, United \\ Kingdom
}

\begin{abstract}
We present a novel multi-dimensional positional encoder approach that uses range-resolved interferometry (RRI) to simultaneously interrogate multiple interferometers differing in optical path length, using only a single photo detector and a single diode laser. Employing only stage-mounted non-polarising beamsplitters and reflections from three orthogonal, externally mounted, planar mirrors, three Cartesian measurement directions are formed, that when combined with measurements from a stage-mounted reference reflection, allow for three-dimensional measurements of stage motion obtained via a single access port. Through a variety of movements of a three-dimensional Piezo-electric stage over its nominal stage working range of $\pm 50 \mu \mathrm{m}$, simultaneous measurements of displacements from three orthogonal dimensions are acquired with a $10 \mathrm{kHz}$ quadrature bandwidth, achieving typical displacement noise densities of below $0.4 \mathrm{~nm} / \sqrt{\mathrm{Hz}}$, with the noise floor dropping to below $0.1 \mathrm{~nm} / \sqrt{\mathrm{Hz}}$ above $50 \mathrm{~Hz}$, as well as high linearity with cyclic errors amplitudes well below $1 \mathrm{~nm}$ in all three directions.
\end{abstract}

Keywords: Displacement Measurement, Range-Resolved Interferometry, Diode Lasers, Dimensional Metrology, Optical Encoder, Interferometry 2020 MSC: 00-02, 78-02, 78-05

\footnotetext{
* Corresponding author

Email address: t.kissinger@cranfield.ac.uk (Thomas Kissinger)
}

Preprint submitted to Optics and Lasers in Engineering

August 4, 2020 


\section{Introduction}

Modern production techniques used to produce nano-scale electronic and optical components require high-precision machinery capable of accurate positioning at nanometre levels. The position measurement techniques employed by these machines must operate at appropriate distances with high resolution and accuracy, where the resolution determines the smallest measurable displacement. Typically they feature positional encoders, utilising optical and non-optical techniques to feed back information about their current position and orientation $[1,2,3,4]$. Non-optical techniques such as piezoresistive sensors $[5,6]$ and capacitive techniques $[7,8,9]$ often require close contact with the target object, and in the case of capacitive sensors, must maintain the angular alignment of electrodes. Restrictions such as these add design complications for many applications and sometimes limit their suitability for manufacturing and production use. Optical encoders utilise pattern-based techniques or laser interferometry to perform displacement measurements. Whilst pattern-based encoders using calibrated pattern strips have been shown to make high-accuracy displacement measurements $[10,11]$, they also require close proximity between the moving stage and the reference frame to operate with accuracy, again severely constraining stage design options. Laser interferometers offer nanometre level resolution capabilities and provide remote, non-contact measurements of the target displacement $[12,13,14,15]$ or, at reduced resolutions, absolute target distance measurements [16]. This generally comes at the cost of increased size and complexity, typically from the interrogation system, as well as various practical drawbacks such as the need, at least for displacement measuring interferometers, to return the target object to a known reference position in the case of startup or beam blockage. Despite these drawbacks, optical interferometry remains an attractive technique for high-precision applications, and as such over the years numerous variations on standard interferometric setups have been developed for metrological purposes in single dimensions [17, 18, 19, 20, 21]. When extended to more than one spatial dimension, however, optical interferometers 


\begin{tabular}{|c|c|c|c|c|}
\hline Sensor Type & Resolution & $\begin{array}{l}\text { Remote } \\
\text { Operation } \\
\text { Possible? }\end{array}$ & $\begin{array}{c}\text { No. of Measured } \\
\text { Degrees of Freedom } \\
\text { per System }\end{array}$ & $\begin{array}{l}\text { Complexity in } \\
\text { Sensing Region }\end{array}$ \\
\hline Piezoresistive & $0.5-10 \mathrm{~nm}$ & No & 1 & 1 sensor \\
\hline Capacitive & $0.5-2 \mathrm{~nm}$ & No & 1 & 1 sensor \\
\hline Standard Interferometer & $0.5-1 \mathrm{~nm}$ & Yes & 1 & 1 mirror \\
\hline Pattern-based Encoder & $0.2-5 \mathrm{~nm}$ & No & 1 or 2 & $\begin{array}{l}1 \text { scale }(1 \mathrm{D} \text { or } 2 \mathrm{D}) \\
\text { and } 1 \text { readout unit }\end{array}$ \\
\hline Grating-based Encoder & $0.1-2 \mathrm{~nm}$ & No & $2[22], 6[26], 6[27]$ & $\begin{array}{l}\text { Various optical elements: } \\
10[22], 13[26], 34[27]\end{array}$ \\
\hline $\begin{array}{c}\text { Range-resolved } \\
\text { Interferometer } \\
\text { (This implementation) }\end{array}$ & $1.3 \mathrm{~nm}$ & Yes & 3 & 3 mirrors, 2 beamsplitters \\
\hline
\end{tabular}

Table 1: Comparison of existing displacement measurement technologies over a $100 \mu \mathrm{m}$ range at $1 \mathrm{kHz}$ bandwidth. Values derived, unless specified, from [1],[3] and [14].

typically require multiple sensing heads or optical access ports. In addition, various designs using gratings to achieve multi-dimensional displacement measurements have been conceived [22, 23, 24, 25, 26, 27, 28].

Table 1 compares a range of approaches to single and multi-dimensional displacement measurements to the approach proposed in this paper, where it can be seen that all compared techniques offer resolutions on the same order of magnitude at nanometer and sub-nanometer levels. However, where these techniques differ is in the complexity of the application, in particular for multidimensional measurements that typically require either highly sophisticated 2D pattern scales or in the case of grating-based techniques, complex setups with numerous, often polarisation-sensitive, optical elements. This has the added effect of increasing cost and typically requiring complex alignment procedures. In addition, existing multi-dimensional approaches lack the ability to operate remotely, where we define remote operation as the ability to work with a wide range of stand-off distances to the moving stage. This allows greater flexibility in stage design or even permits operation through windows, for example a vacuum window. It can be seen from Table 1 that there is a need for lowcomplexity, remotely operating multi-dimensional approaches and in this paper we present a proof-of-concept experiment and results demonstrating a novel, 
three-dimensional interferometric optical encoder with significant advantages over the state-of-the-art, utilising only a single optical beam entering through a single optical access port. Together with a very simple optical setup consisting of two stage-mounted beamsplitters and three static mirrors without the use of any polarisation-sensitive components, this setup allows the simultaneous acquisition of displacement measurements in three orthogonal dimensions. This is achieved using the range-resolved interferometric (RRI) technique [29] to measure interferometric phase changes at multiple locations along a single or multiple folded optical beam paths. RRI utilises optical wavelength modulation resulting from sinusoidal injection current modulation of a continuous-wave laser diode to interrogate multiple interferometers using a single laser source and photo detector. RRI examines the superimposed interference pattern created by the multiplexing of all constituent interferometers within the beam path and distinguishes them based on their optical path differences (OPDs). In addition to fibre-optic strain[30] and shape sensing[31], we have previously applied RRI to multiple-surface laser vibrometry[32] which allows movements of multiple semi-transparent surfaces along a single optical beam to be interrogated simultaneously for advanced vibration analysis. Here, we present results demonstrating the use of this technique as a three-dimensional stage encoder with regards to various stage motions along with a detailed analysis of its noise and linearity performance, significantly extending our initial work on a two-dimensional encoder[33]. It is thought that the simplicity and space-effectiveness of this approach, as well as the nanometre-level displacement resolution and linearity performance achievable, along with the ability for remote interrogation, offers an attractive alternative to existing methods and could lead to new developments in cases where the limitations of existing encoder techniques are an obstacle to implementing novel nanopositioning stage applications. 


\section{Principles and Methods}

The proposed method uses the multiplexing of several interferometers that differ in OPD to implement a three-dimensional encoder, therefore careful selection of the OPDs of the interferometers of interest is required to avoid overlap between desired and parasitic constituent interferometers which can be the cause of cyclic errors. As with all interferometric techniques, cyclic errors [34], i.e. periodic non-linearities with a period corresponding to 0.5 or 1 interrogation wavevectors, will occur in phase measurements if signal contributions from interferometers created by parasitic and secondary reflections are present. As a result, when developing an optical setup for interferometric purposes the placement of elements within the setup is critical. Unlike conventional interferometry, RRI allows for the visualisation of these parasitic interferometers and, through appropriate spacing, provides methods to prevent their overlap to avoid cyclic errors. Furthermore, it is important to note that RRI, unlike most interferometric techniques, does not rely on polarisation splitting and is therefore immune to polarisation leakage, which is often the dominant cause of cyclic errors in interferometry[35].

In order to demonstrate the benefits of using the range-resolved interferometric technique as a three-dimensional encoder, a simple proof-of-principle optical setup was conceived. Two beamsplitter cubes are mounted on a threeaxis motion stage, surrounded on three sides by orthogonal planar mirrors. On a fourth side a fibre collimator is mounted which constitutes the single access port required by this technique. The beamsplitters and mirrors are positioned such that the laser beam incident on the first beamsplitter cube will split to illuminate the second beamsplitter and one mirror, with the beams from the second beamsplitter illuminating the two remaining mirrors. All three mirrors reflect the beams back to the fibre collimator via the same optical paths and the re-coupled light is guided back to the signal processing hardware. By interrogation of the interferometers created between each mirror and the fibre tip, and by numerically subtracting from this the displacement of a common reference 
interferometer created between the fibre tip and the first incident surface of the beamsplitter, $\mathrm{R}$, stage displacements in the three orthogonal dimensions can be interrogated simultaneously. A schematic of this setup is shown in Fig. 1 in addition to a $3 \mathrm{D}$ representation.

As illustrated in Fig. 1, the reflections between each mirror $\mathrm{M}_{\mathrm{x}}, \mathrm{M}_{\mathrm{y}}$ and $\mathrm{M}_{\mathrm{z}}$ and the Fresnel reflection from the flat-polished fibre tip then form the desired interferometers spanning the geometrical lengths $\alpha, \beta$ and $\gamma$, respectively. $\varphi$ corresponds to the path length of undesired interferometers between any adjacent pair of beamsplitter surfaces which overlap due to identical optical path differences, while signals corresponding to interferences between non-adjacent beamsplitter surfaces or multiple bounces are also present at integer multiples of $\varphi$. Also significant is the interferometer between the reference surface denoted as R, i.e. the front surface of the first beamsplitter cube, and the fibre tip, with its geometrical distance denoted as $\delta$ in Fig. 1. Fig. 1(d) then shows an illustration of the expected interferometric signal strength as a function of OPD, where peaks corresponding to undesired interferometers are drawn in black. 


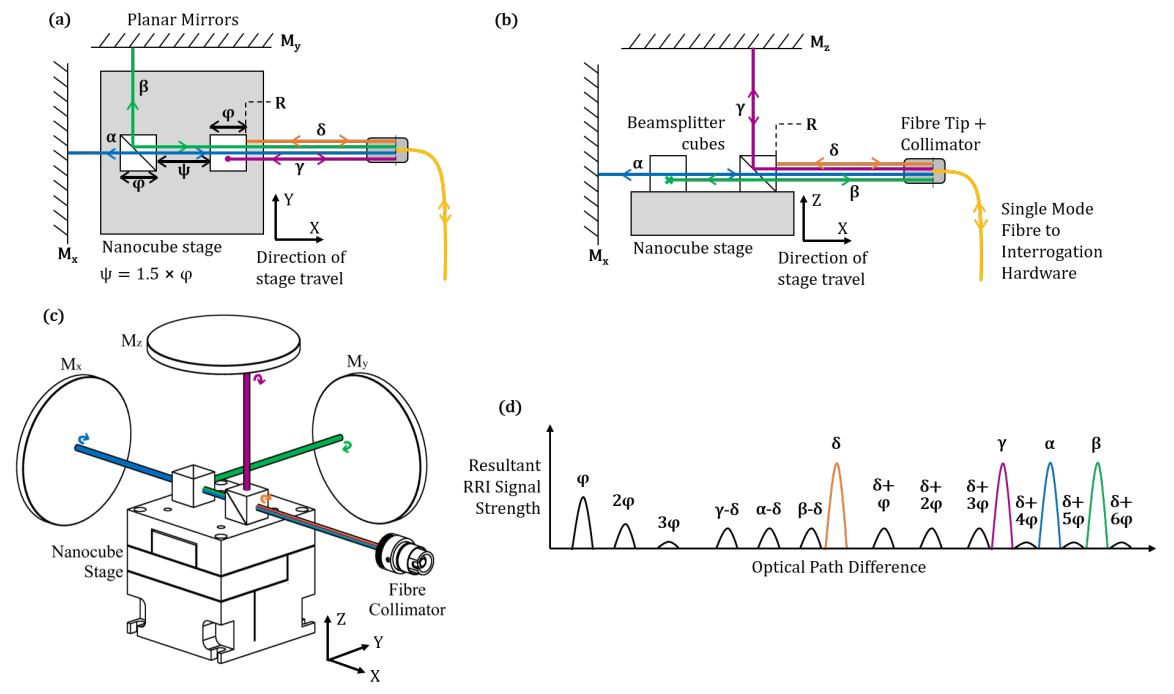

Figure 1: Schematic of the optical setup. (a) shows the top-down view with the relevant geometrical distances highlighted in addition to the reference surface R. (b) shows the sideview with the relevant geometrical distances highlighted. (c) shows a 3D view of the encoder including the orientation of the Nanocube stage. The OPDs of the constituent interferometers arising from this setup are illustrated in (d) with the parasitic interferometers drawn in black. In all sub-figures, distances $\alpha, \beta$ and $\gamma$ correspond to the interferometers created between the $\mathrm{X}, \mathrm{Y}$ and $\mathrm{Z}$ mirrors, labelled as $\mathrm{M}_{\mathrm{x}}, \mathrm{M}_{\mathrm{y}}$ and $\mathrm{M}_{\mathrm{z}}$, respectively, interfering with the Fresnel reflection from the flat-polished fibre-tip. $\delta$ refers to the distance of the reference interferometer between the first incident surface of the first beamsplitter, the reference surface $\mathrm{R}$, and the fibre tip, while $\varphi$ corresponds to path lengths of the undesired interferometers between any adjacent pair of beamsplitter surfaces.

In the proposed approach, the mirrors and beamsplitters are positioned such that the OPDs of the desired interferometers are unique to the OPDs of all other constituent interferometers including those formed by secondary reflections, where any overlap of the OPDs of the desired interferometers with those caused by secondary reflections would cause cyclic errors in the measurements. First, by maintaining a sufficiently large distance between the fibre tip and the stage, the desired interferometers $\alpha, \beta, \gamma$ and $\delta$ that arise from interference between the mirrors $\mathrm{M}_{\mathrm{x}}, \mathrm{M}_{\mathrm{y}}$ and $\mathrm{M}_{\mathrm{z}}$ or the reference surface $\mathrm{R}$ with the fibre tip are offset from undesired interferometers of path lengths $\gamma-\delta, \beta-\delta$ and $\alpha-\delta$ 
between the reference surface $R$ and the mirrors $M_{x}, M_{y}$ and $M_{z}$. Furthermore, the mutual interferences between any two beamsplitter surfaces occurring at multiples of $\varphi$ can be seen on the left of Fig. 1(d), where the two beamsplitters themselves are placed a fixed distance $\psi$ apart such that $\psi$ approximately equals the in-air equivalent $\mathrm{OPD}$ of $\varphi$, i.e. $\psi=1.5 \varphi$. The mirrors $\mathrm{M}_{\mathrm{x}}, \mathrm{M}_{\mathrm{y}}$ and $\mathrm{M}_{\mathrm{z}}$ are then positioned such that the OPDs of the respective interferometers $\alpha, \beta$ and $\gamma$ between the mirrors and the fibre tip lie between the optical path differences created between the fibre tip and the beamsplitter surfaces and multiple passes thereof $(\delta+\mathrm{n} \varphi$ with any positive integer $\mathrm{n})$. Furthermore, there may also be higher order parasitic interferometers caused by multiple bounces between reflecting surfaces. To suppress these, a weakly focused beam is used, where the beam curvature causes increasingly diminishing return light levels from multiple reflections within the system, rendering contributions to the interferometric signal from these reflections negligible.

\section{Experimental Work}

\subsection{Interrogation Hardware}

To interrogate this optical setup a single Eblana Photonics discrete-mode laser diode [36] (EP1512-DM-B, power $\approx 5 \mathrm{~mW}$, linewidth: $\approx 1 \mathrm{MHz})$ has its emission wavelength sinusoidally modulated by injection current modulation at a frequency of $24.4 \mathrm{kHz}$, with a wavelength modulation amplitude of $\pm 0.31 \mathrm{~nm}$ around its centre wavelength of $1520.08 \mathrm{~nm}$ (in air), as measured using an optical spectrum analyser (Yokogawa AQ6370C). For this particular diode this is close to the maximum possible amplitude, obtained by modulating from just above the laser threshold to the maximum current. As shown in Fig. 2(a) the emitted light is guided by a fibre-optic circulator, via a single-mode fibre lead, to the collimator and optical setup shown in Fig. 1. The returning light is then guided by the circulator to an InGaAs photo detector and the resulting interferometric signals are acquired by an analogue-to-digital converter (ADC) with a sample rate of $100 \mathrm{MHz}$ and are demodulated in real time by field programmable gate 
array (FPGA) based signal processing hardware. Measurements were recorded as raw phase data, and converted to displacements in post-processing using the measured central evaluation wavelength of $1520.08 \mathrm{~nm}$. A picture of the fully-enclosed and portable interrogation unit can be seen in Fig. 2(b).

(a)

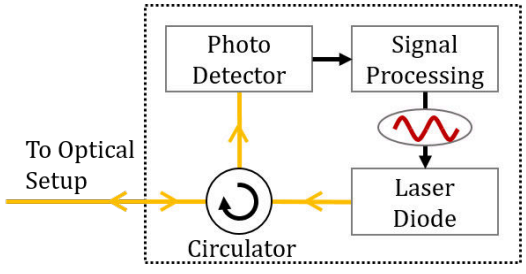

(b)

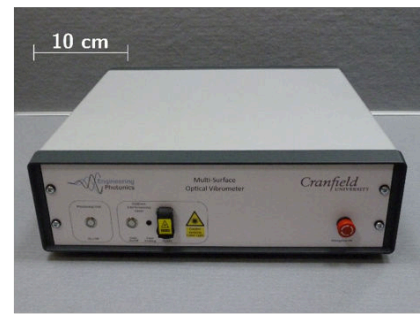

Figure 2: The interrogation hardware used in this system, with (a) showing the interrogation hardware setup, linking to the optical setup illustrated in Fig. 1, and (b) showing a picture of the fully-enclosed enclosed interrogation unit.

\subsection{Optical Setup}

As described earlier and shown in Figs. 1(a) and (b), a simple optical setup is used in this proof-of-concept experiment. Three planar aluminium mirrors are mounted on orthogonal faces of a Thorlabs $60 \mathrm{~mm}$ cage cube within stackable lens tubes. On an opposing side of the cube, a weakly focusing collimating lens is mounted within a 3-axis kinematic mount which allows for adjustment of the optical beam axis, comprising the single optical access port required by this setup. The beam is weakly focused with a focus location approximately in front of the first beamsplitter surface to reduce contributions in the interferometric signal from multiple passes through the optical system interfering with other reflections. The beam has a Gaussian divergence of $0.13^{\circ}$, which we estimate will cause a maximum error of $0.12 \mathrm{~nm}$ over the travel range of the stage [37], which is negligible. Within the confines of the cube, a Physik Instrumente Nanocube stage (P-611.3) with a travel range of $\pm 50 \mu \mathrm{m}$ in three Cartesian dimensions is centrally positioned on top of a 2-axis goniometer and rotation mount. This hardware allows for alignment of the Nanocube stage to the axes of the cage cube and is shown schematically in Fig. 3 alongside a photo of the 
complete optical assembly of the encoder.

(a)

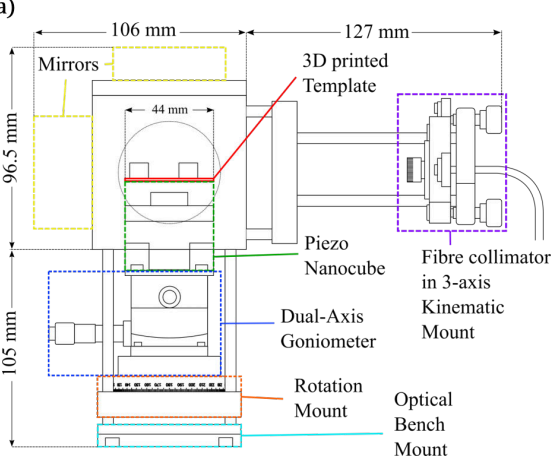

(b)

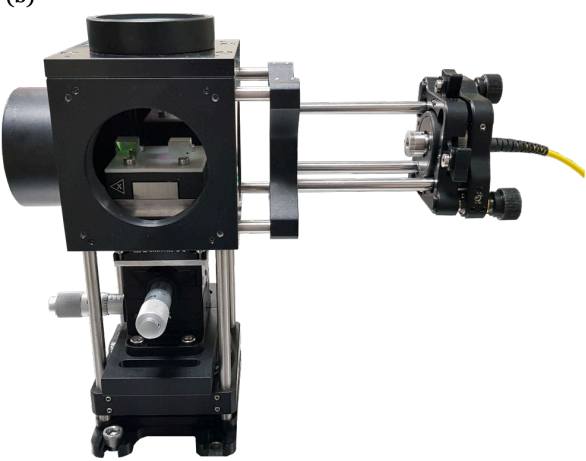

Figure 3: (a) shows a schematic of the optical setup of the encoder with some of the key alignment hardware highlighted and the main dimensions of the encoder labelled. (b) shows a photo of the complete optical setup.

The stage utilises internal strain gauge sensors to feedback position to the stage controller, allowing for controlled, high accuracy movements. On the surface of the stage, two beamsplitter cubes of $10 \mathrm{~mm}$ cube length are mounted within a 3D-printed template, designed to position the beamsplitters square to one another and with a fixed separation ( $\psi$ in Fig.1(a)) of $15 \mathrm{~mm}$ along the optical axis. The front surface of the first beamsplitter acts as reference surface R. This surface has been polished to remove the anti-reflection coating in order to yield a strong Fresnel reflection to be used as a reference interferometer, however the other beamsplitter faces still have the original anti-reflective coatings left in order to suppress contributions of multiple reflections between beamsplitter faces.

Fig. 4(a) shows an example of the interferometric signal measured by the photo detector over one modulation period for this setup, overlaid with the window function used in the RRI signal processing[29]. It can be seen that strong intensity modulation is present in the signal, however, this can be readily compensated by the RRI processing algorithms. A plot of the RRI signal power against the demodulation phase carrier amplitude, measured in radians and ap- 

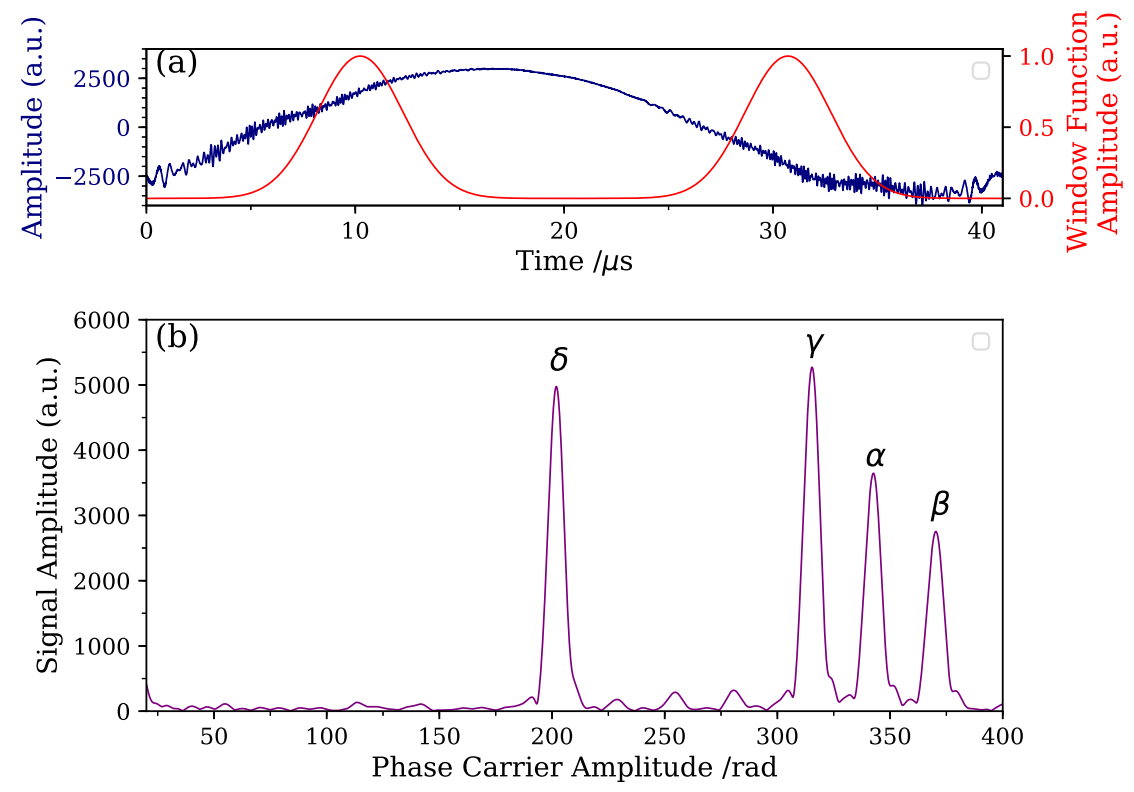

Figure 4: RRI processing graphs: (a) shows the raw interferometric signal and the Gaussian windows applied in the demodulation process on the secondary y-axis. (b) shows a graph of the range-resolved signal power against the demodulation phase carrier amplitude, approximately proportional to the optical path difference, where the same notation for the desired interferometers $\alpha, \beta, \gamma$ and $\delta$ introduced in Fig. 1(d) has been used.

proximately proportional to OPD, can be calculated from the interferometric signal shown in Fig. 4(a) and is shown in Fig. 4(b). This plot shows a tomographic view of all constituent interferometers present in the system and can be used to diagnose the optical configuration, for example the presence of multiple reflections, as well as aid the process of optical alignment through maximisation of the desired peak heights. With the present wavelength modulation amplitude of $0.31 \mathrm{~nm}$, the scaling factor in Fig. $4(\mathrm{~b})$ is approximately $0.58 \mathrm{~mm} / \mathrm{rad}$ for the in-air equivalent path distances of the constituent interferometers. For example, it can be seen that reference surface $\delta$ is placed at $\approx 117 \mathrm{~mm}$ distance from the fibre tip and that the full-width half maximum width of the peaks is 8.4 rads or $4.9 \mathrm{~mm}$, which also approximately corresponds to the minimum separation between adjacent signal sources that needs to be maintained in or- 
der to avoid cyclic errors due to peak overlap. This plot also labels the desired interferometers $\alpha, \beta, \gamma$ and $\delta$ corresponding to Fig. 1(d). These four interferometers are then selected for real-time phase demodulation in the FPGA, where the current interrogation system can simultaneously demodulate up to 24 range channels. The desired displacements in $\mathrm{X}, \mathrm{Y}$, and $\mathrm{Z}$ direction are then obtained by numerically subtracting the respective displacements corresponding to interferometers $\alpha, \beta$ and $\gamma$, respectively, from the displacement of the reference surface $\mathrm{R}$ obtained from interferometer $\delta$.

\section{Results}

In order to demonstrate the measurement capabilities of this concept, threedimensional displacement measurements over a variety of stage movements, including linear and helical motions, were conducted and recorded with an interferometric quadrature bandwidth of $10 \mathrm{kHz}$. Additionally, a characterisation of the noise and linearity performance is carried out.

\subsection{Linear Motion Measurements}

In the first experiment, starting from a central position, the stage was moved to a fixed offset in the X-direction, then swept to the same but negative offset in the X-direction before being returned to the central position. The same procedure is then repeated in the Y-direction followed by the Z-direction with displacements in the three directions simultaneously acquired. In Fig. 5(a), the stage is moved according to this procedure with an amplitude of $\pm 50 \mu \mathrm{m}$ and with a linear velocity of $10 \mu \mathrm{m} / \mathrm{s}$. Fig. 5 (b) shows a restricted range of the same data around the zero-displacement axis in order to better see any undesired displacements measured in the off-motion axes. Fig. 5(c) shows the displacements measured in $\mathrm{X}, \mathrm{Y}$ and $\mathrm{Z}$ for the similar motion patterns as described previously, but with an amplitude of $\pm 5 \mu \mathrm{m}$ and with stage axis velocities of $1 \mu \mathrm{m} / \mathrm{s}$. Fig. 5(d) again shows the same data used in Fig.5(c) but with a restricted range around the zero displacement axis. Fig. 5(e) shows X, Y and Z displacements 
for a similar motion with an amplitude of $\pm 0.5 \mu \mathrm{m}$ and with a velocity of 0.1 $\mu \mathrm{m} / \mathrm{s}$, with Fig. 5(f) again showing a restricted range of the same data set. It can be seen in Figs. 5(a), (c) and (e) that the displacement measurements for movement in all three directions can be readily obtained in all three cases covering large, medium and small commanded motions, demonstrating the ability of this system to measure in multiple displacement directions simultaneously. Furthermore, it can be observed in Figs. 5(b), (d) and (f) that in addition to the commanded motion there are displacement signals present in the off-axis directions. For motion in the $\mathrm{X}$ direction, this occurs primarily in the $\mathrm{Y}$ direction at an amplitude of approximately $1.2 \%$ of the $\mathrm{X}$ direction amplitude, while the $\mathrm{Z}$ direction exhibits only minimal off-axis displacements. For motion in the $\mathrm{Y}$ direction, only minimal off-axis displacements are observed in both the $\mathrm{X}$ and the $\mathrm{Z}$ directions. For $\mathrm{Z}$ motion, the overall largest off-axis displacements can be observed, reaching approximately $2.0 \%$ and $1.8 \%$ of the stage motion amplitude for the $\mathrm{X}$ and $\mathrm{Y}$ directions, respectively.

The observed off-axis displacements could be caused by three reasons. 1) Non-flatness of the mirrors $\mathrm{M}_{\mathrm{x}}, \mathrm{M}_{\mathrm{y}}$ and $\mathrm{M}_{\mathrm{z}}$. 2) Genuine crosstalk in the stage motion. 3) Misalignment of the optical axes relative to the stage motion directions. While neither the first nor the second reason can be ruled out and both may also contribute to the observed off-axis displacements, the dominant cause is most likely the misalignment of the optical axes to the stage motion direction. This is because in this proof-of-principle experiment the beamsplitters were only very coarsely aligned using the $3 \mathrm{D}$ printed template that holds the beamsplitters in place. Adjustment elements for the beamsplitters were too difficult to incorporate in the setup due to the space-constraint environment on the stage top plate. With no adjustments on the 3D printed template possible, the stage was then aligned using the goniometers and the rotation stage to optimise the amplitude in the return signals in Fig. 4(b), where generally maximum signal amplitudes would be obtained at normal incidence to the mirror. If the beamsplitters are misaligned relative to the stage principle axes, this alignment procedure would then cause the stage motion axes to be misaligned 
with the optical axes of the measurement beams and could explain the observed off-axis displacements. As the $\mathrm{Z}$ direction is only dependent on the orientation of a single beamsplitter placed flat on the stage top plate, this direction is automatically aligned to the stage principle axis and therefore less prone to off-axis displacements and it can be seen in all subplots of Fig. 5 that the $\mathrm{Z}$ axis displacement signal remains flat during the movement of the other axes. Conversely, as the $\mathrm{Y}$ direction is dependent on the orientation and alignment of both beamsplitters in all three planes, we expect it to be the most difficult direction to align and indeed it can be observed that the $\mathrm{Y}$ direction exhibits the largest overall off-axis displacements. In addition to the discussed alignment errors, drifts in the stage zero position of up to $30 \mathrm{~nm}$ can also be observed in Fig. 5(f). Drifts in the zero position could be explained through changes of the laser wavelength or the air refractive index as well as through non-ideal stage behaviour, such as thermal stage expansion or angular stage errors. In general, laser wavelength or air refractive index changes would cause drifts that are proportional to the optical path length, however, such a proportionality cannot be observed in Fig. 5(f), therefore non-ideal stage behaviour is the more likely cause and further investigation is needed to confirm this. 

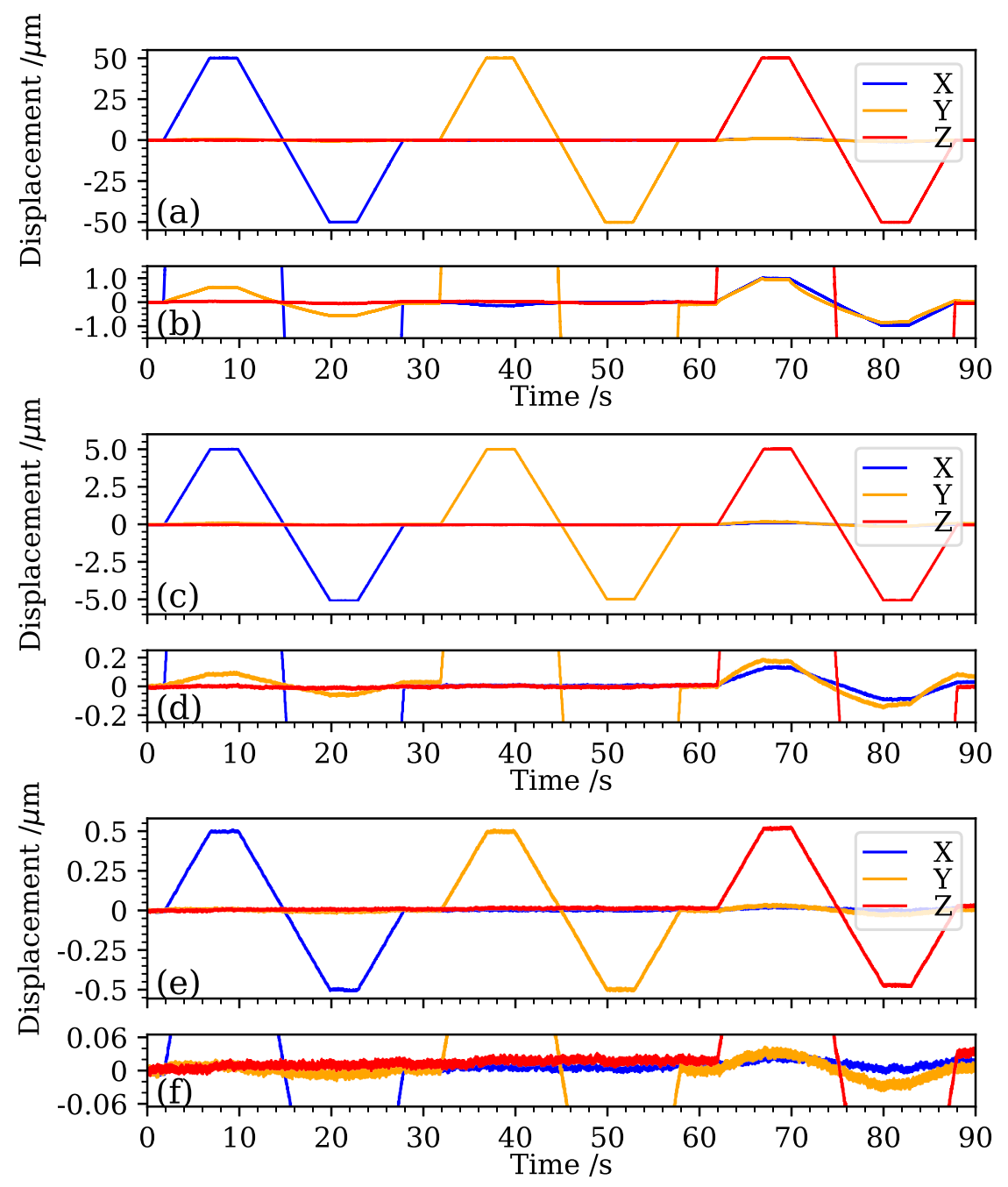

Figure 5: Simultaneous measurements of $\mathrm{X}, \mathrm{Y}$ and $\mathrm{Z}$ displacements for motion in the $\mathrm{X}$ direction of the stage followed by a similar motion in the $\mathrm{Y}$-direction and Z-direction. (a) shows a linear movement across the total range of the stage, $\pm 50 \mu \mathrm{m}$, at a speed of $10 \mu \mathrm{m} / \mathrm{s}$, while (b) shows a closer view of the same results over a smaller displacement range around the zero position. (c) and (d) plot the results in a similar fashion to (a) and (b) but for a smaller stage movement across $\pm 5 \mu \mathrm{m}$ at velocities of $1 \mu \mathrm{m} / \mathrm{s}$. (e) and (f) then show similar plots for $\pm 0.5 \mu \mathrm{m}$ movements at velocities of $0.1 \mu \mathrm{m} / \mathrm{s}$. 


\subsection{Stage Encoder Comparison}

To provide an independent verification of the interferometric data, we can compare the measured displacements to the strain gauge sensors integrated within the stage. In this experiment, for each direction, the stage is moved in a repeated triangular pattern of $0.2 \mathrm{~Hz}$ frequency and an amplitude of \pm 15 $\mu \mathrm{m}$. The displacements for both the stage and the encoder are recorded and the results are compared in Fig. 6.
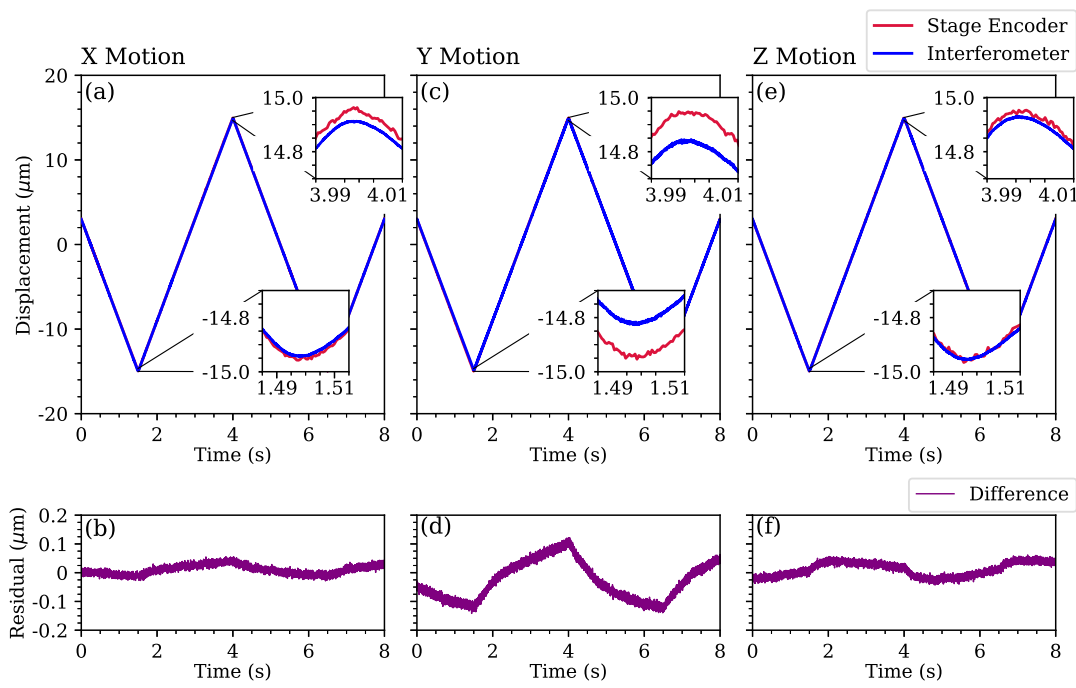

Figure 6: (a) shows the measured displacement from the interferometer and from the integrated stage sensor of a $\pm 15 \mu \mathrm{m}$ triangular motion in the $\mathrm{X}$ direction, while (c) and (e) show the corresponding data for the $\mathrm{Y}$ and $\mathrm{Z}$ directions, respectively. For all three directions the calculated differences between stage encoder and interferometer are plotted below the respective graphs in (b), (d) and (f).

In general it can be seen in Fig. 6 that there is good agreement between the stage encoder and the measured interferometric data, with maximum errors below $\pm 0.15 \mu \mathrm{m}$ or $1 \%$ of the travel distance for motion in the Y-direction and below $0.5 \%$ for motion in the $\mathrm{X}$ and $\mathrm{Z}$ directions. A general error proportional to the travel displacement can be attributed to a mismatch in scaling factors in either the stage encoder or the interferometer, where errors in the interferometer can be caused by wavelength or environmental refractive index uncertainties. 
Furthermore, as discussed previously, the remaining errors are likely to be a result of misalignment of the optical axes to the stage motion axes. Here, because the alignment of the $\mathrm{Y}$ direction depends on the precise alignment of two beamsplitters, the increase of the error amplitude in the $\mathrm{Y}$ direction compared to the $\mathrm{X}$ and $\mathrm{Z}$ direction axes seems plausible. Additionally, the superior noise performance of the interferometric data compared to the in-built strain gauge encoders that exhibit an instantaneous noise standard deviation of $6.3 \mathrm{~nm}$ is also visible in insets in Figs. 6(a), (c) and (e).

\subsection{Noise and Linearity Measurements}

In addition to demonstrating the simultaneous measurement of displacements in multiple dimensions, this arrangement can be used to estimate the typical noise levels achievable with this interrogation technique. As an example, we have conducted an experiment with a sinusoidal movement in one direction (X) and no movement in the other directions ( $\mathrm{Y}$ and $\mathrm{Z}$ ). For a sinusoidal motion in the $\mathrm{X}$-axis of the stage, with a frequency of $5 \mathrm{~Hz}$ and an amplitude of $0.5 \mu \mathrm{m}$, measurements of the interferometers for each dimension and of the reference interferometer were acquired over a period of $50 \mathrm{~s}$. The measurements were then sliced into $1 \mathrm{~s}$ long segments and the amplitudes of the Fourier transform were averaged to obtain an estimate of the displacement amplitude spectral density.

Figure 7(a) shows one $1 \mathrm{~s}$ long section of the measurement for directions $\mathrm{X}$, $\mathrm{Y}$ and $\mathrm{Z}$ when the stage was directed to perform a pure sinusoidal motion in the $\mathrm{X}$ direction. Figure $7(\mathrm{~b})$ then shows the corresponding displacement amplitude spectral densities for the three measurement signals $\mathrm{X}, \mathrm{Y}$ and $\mathrm{Z}$ along with the

direct measurement of interferometer $\alpha$, which as can be seen in Fig. 1(d) is stationary regardless of the stage motion and mechanical stage vibrations and thus gives a direct estimate of the intrinsic noise due to the interrogation system. In Fig. 7(b) we can see a peak at $5 \mathrm{~Hz}$ from the motion of the stage the $\mathrm{X}$ direction at $0.50 \mu \mathrm{m}$ amplitude, as expected. A off-axis displacement of $0.01 \mu \mathrm{m}$ amplitude is observed in the $\mathrm{Y}$ direction, which corresponds to a ratio of $2.0 \%$, the same ratio that was previously observed in Fig. 5. The $\mathrm{Z}$ direction exhibits 

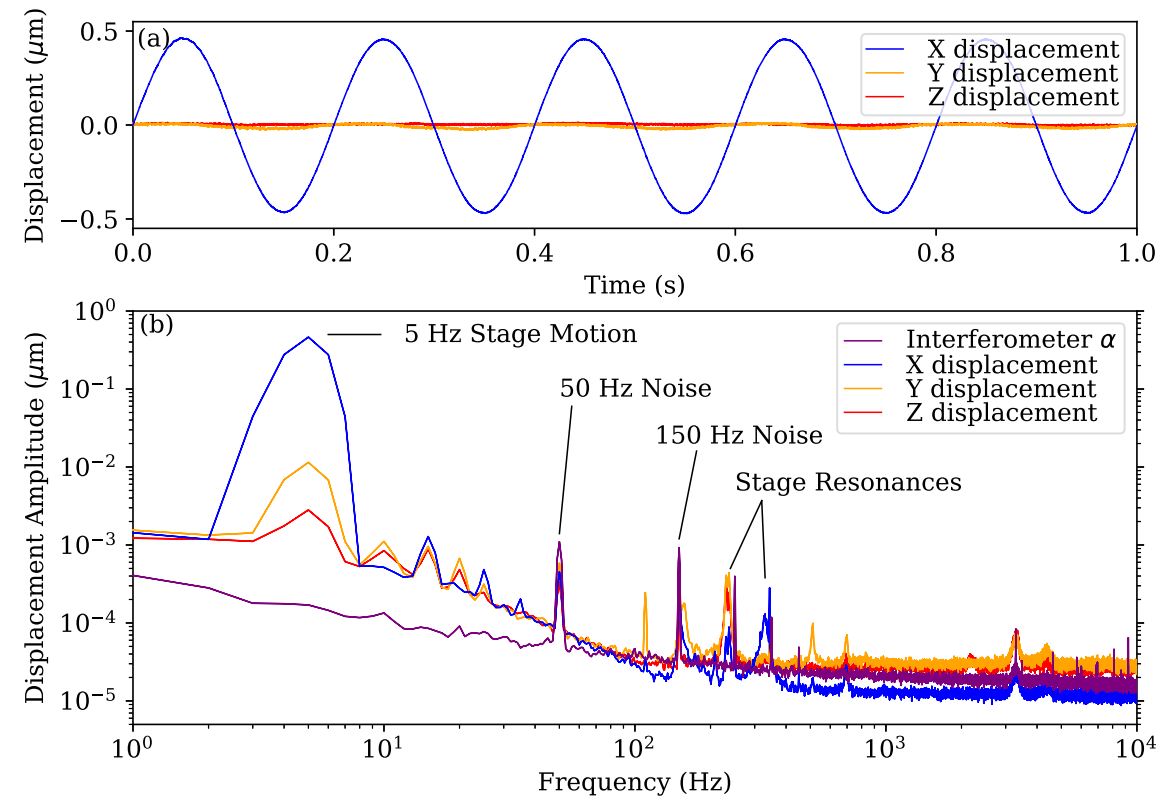

Figure 7: (a) shows displacement data for $\mathrm{X}, \mathrm{Y}$ and $\mathrm{Z}$ for a simple sinusoidal motion of \pm 5 $\mu \mathrm{m}$ movement in the X-direction. (b) shows the calculated displacement amplitude spectral density estimate over a $1 \mathrm{~s}$ bandwidth for the measured X, Y and Z directions. Additionally, the direct measurement of interferometer $\alpha$ in Fig. 1(d) without the subtraction of the reference interferometer $\delta$, is shown for comparison, where this signal is stationary regardless of stage movement and is thus a direct measure of the instrumental noise.

a much smaller off-axis displacement amplitude of $0.003 \mu \mathrm{m}$, corresponding to a $0.6 \%$ ratio. By comparison with the instrumental noise obtained from the spectrum of the direct measurement of interferometer $\alpha$, it can be seen that below $100 \mathrm{~Hz}$ the measurements in the $\mathrm{X}, \mathrm{Y}$ and $\mathrm{Z}$ directions exhibit increased noise contributions which are likely due to the vibration sensitivity of the mechanical setup used for stage alignment (the rotation stage and goniometers). This increased vibration sensitivity is likely to result in increased noise contributions in this frequency region from ambient excitations of the mechanical structure. Conversely, the instrumental noise given by the measurement of interferometer $\alpha$ is free from these mechanical noise components and only exhibits $1 / \mathrm{f}$ noise typical for interferometric systems and generally stays below a displacement 
noise level of $0.4 \mathrm{~nm} / \sqrt{\mathrm{Hz}}$. The complete lack of discernible contributions from the stage movement to the spectrum of interferometer $\alpha$ also confirms that any instrumental cross-talk between constituent interferometers is below a level of $0.04 \%$. In addition to instrumental noise caused by the electricity supply at $50 \mathrm{~Hz}, 150 \mathrm{~Hz}$ and $250 \mathrm{~Hz}$, present in all four signals, various spectral features between $150 \mathrm{~Hz}$ to $300 \mathrm{~Hz}$ can be found for the three stage directions X, Y and $\mathrm{Z}$ but not for the signal of interferometer $\alpha$. These are thought to be stage resonances and approximately match the mechanical resonance frequencies given in the stage data sheet[38]. In general, in most regions above $100 \mathrm{~Hz}$, the displacement amplitude spectra for all four signals drops to noise floor levels between $0.01 \mathrm{~nm} / \sqrt{\mathrm{Hz}}$ and $0.04 \mathrm{~nm} / \sqrt{\mathrm{Hz}}$. Over a typical $1 \mathrm{kHz}$ bandwidth for the case of the interferometer $\alpha$ without influence of mechanical vibrations, this represents a resolution $(1 \sigma)$ of $1.3 \mathrm{~nm}$ achievable using this technique.

To characterise the linearity achievable with this experimental setup, the stage is set to perform a linear motion at a given velocity such that any cyclic errors will occur at a fixed frequency within the frequency region from $50 \mathrm{~Hz}$ to $100 \mathrm{~Hz}$, which, as can be seen in Fig. 7(b), is generally flat and free from spectral features. To place the frequency of any cyclic errors within this frequency region, the stage is sequentially moved in a triangular motion in each direction with a velocity of $50 \mu \mathrm{m} / \mathrm{s}$ and the displacement in the relevant direction is measured. In order to remove any non-ideal motion effects at the turning points from the analysis, a $1 \mathrm{~s}$ long segment over the central portion of the ramp is selected and a linear fit subtracted. Then a polynomial fit is fitted to this data to remove any remaining effects due to non-uniform motion. The section of the triangular displacement signal used for this analysis process is also illustrated in Fig. 8(a). The displacement amplitude spectral density is then calculated in a similar way as in Fig. 7(b), using 20 repeats of this data. For a velocity of $\mathrm{v}=50.0 \mu \mathrm{m} / \mathrm{s}$, at a centre wavelength of $\lambda=1520.08 \mathrm{~nm}$, we expect first order cyclic errors at a frequency of $2 v / \lambda \approx 65.8 \mathrm{~Hz}$, where the factor of two is because the interferometer operates in reflection. To confirm that any errors at this frequency are cyclic errors, a second set of measurements are performed 
with an increased velocity of $70 \mu \mathrm{m} / \mathrm{s}$, which should shift the frequency of any first order cyclic errors to $\approx 92.1 \mathrm{~Hz}$.

Fig. 8(a) shows an example period of the measured triangular displacement, and the $1 \mathrm{~s}$ section of the ramp used in this analysis. From Fig.8(b) and (c) we can see peaks corresponding to the $50 \mathrm{~Hz}, 150 \mathrm{~Hz}$ and $250 \mathrm{~Hz}$ mains harmonics and spectral features due to mechanical resonances of the stage at $175-180 \mathrm{~Hz}$ in the Y-direction and between $220-240 \mathrm{~Hz}$ in the $\mathrm{X}$ and $\mathrm{Z}$ directions that were also present in Fig. 7(b). As these features remain static between the two experiments they cannot be caused by cyclic errors as different velocities are used. In Fig. 8(b), for all three directions, we can also see a peak corresponding to the expected first order cyclic error cyclic errors at $65.8 \mathrm{~Hz}$, while in Fig. 8(c) we can observe that for all three direction this peak has moved to the frequency of $92.1 \mathrm{~Hz}$ expected for this velocity. This shift in frequency clearly indicates that these peaks are due to cyclic errors. It can be seen in Figs. 8(b) and (c) that in all cases, the displacement amplitudes of the cyclic errors remain well below $1 \mathrm{~nm}$, while furthermore the complete absence of second-order cyclic errors at double the respective first-order cyclic error frequencies is also evident in Figs. 8(b) and (c). Although, as discussed previously, suppression of secondary reflections and multiple bounces is achieved through weak focusing of the laser, we expect that the remaining cyclic errors occur as a result of secondary reflections within the beam path interfering with straight reflections such that the OPD between them is the same or close to the OPD of the interferometer of interest. Here future, more refined implementations of the proposed concept might be able to improve this further. A $1 \mathrm{~nm}$ cyclic error amplitude is approximately equal to a phase error of $0.5^{\circ}$ and the lowest cyclic error achieved for the $\mathrm{Z}$ direction in Fig. 8(b) is as low as $0.15 \mathrm{~nm}$ or $0.07^{\circ}$. Therefore the achieved results are comparable with respect to linearity performance to highly sophisticated interferometric setups, where typical cyclic errors range between $0.9^{\circ}$ and $0.05^{\circ}[35]$, however, the results achieved here are realised using a much simpler optical setup without polarisation-sensitive components. 

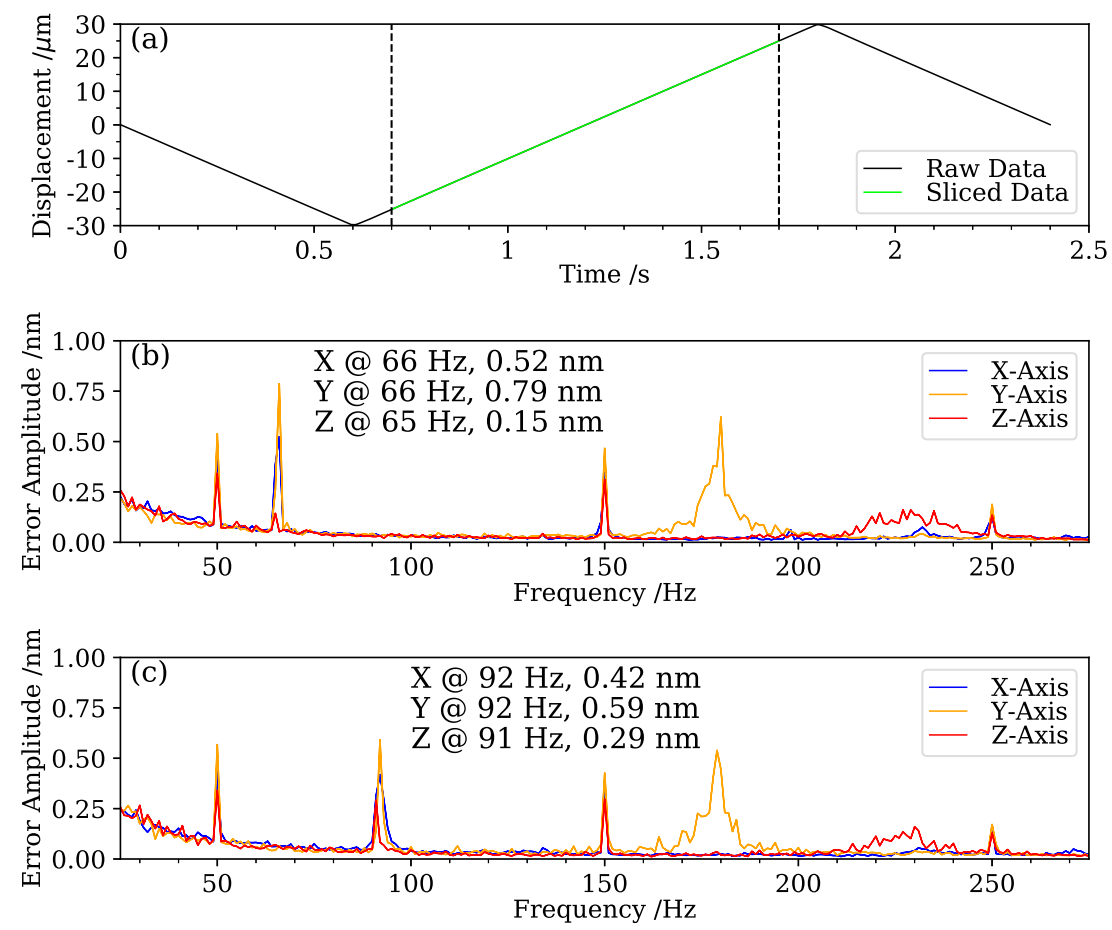

Figure 8: (a) shows an example of one period of one of the triangular motion measurements used in this experiment and highlights the $1 \mathrm{~s}$ analysis region evaluated. (b) shows the displacement noise spectral density estimate for a $50 \mu \mathrm{m} / \mathrm{s}$ movement, while (c) shows this for a $70 \mu \mathrm{m} / \mathrm{s}$ movement. In both (b) and (c) the determined cyclic error contributions occur at different frequencies and their extracted displacement amplitudes are labelled.

\subsection{Helix Measurements}

Measurements were also performed of simultaneous three-dimensional motion. In addition to circular motion in a plane, created by sinusoidal and cosinusoidal motion in the respective axes, a linear stage motion is also applied in the third Cartesian direction, creating a helical motion. Fig. 9(a) shows the measured displacements plotted in 3D for an $\mathrm{X}-\mathrm{Y}$ circular motion combined with a linear motion in Z. Also of interest is how the measured displacements are projected into the plane normal to the linear motion as the stage moves. For the $\mathrm{X}-\mathrm{Y}$ circular motion, the amplitude of the measured displacement is plotted 
in polar representation in Fig. 9(b). The same motion is then performed with a $\mathrm{Y}-\mathrm{Z}$ circular motion and a linear motion in the $\mathrm{X}$-direction, with the measured displacements shown in Fig. 9(c), and the polar representation of the Y-Z plane measurements shown in Fig. 9(d). A third helix was performed with an X-Z circular motion, and a linear motion in Y. The measured displacements for this motion are shown in Fig. 9(e) in 3D representation, with a polar representation of the measured displacement amplitudes in the X-Z plane shown in Fig. 9(f).

As demonstrated by Figs. 9(a), (c) and (e), this technique has the ability to interrogate and measure three dimensions simultaneously and independently, even during complex motion patterns. The projections of the helical motion in the plane normal to the linear motion shown in Figs. 9(b),(d) and (f) should ideally resemble concentric circular displacements due to the commanded circular motion pattern. However, in reality "footprint" patterns are observed that deviate from the ideally circular displacements and, in the case of Figs. 9(b) and (d), are not concentric. The likely cause of the observed non-concentricity of these patterns are the previously discussed misalignments seen in Fig. 5. For example, it can be seen in Fig. 5 that for a motion in the $\mathrm{Y}$ direction, there are only negligible off-axis displacements in the $\mathrm{X}$ and $\mathrm{Z}$ direction, i.e. both the $\mathrm{X}$ and the $\mathrm{Z}$ mirrors are parallel to the $\mathrm{Y}$ motion axis. Hence, for the helix with the linear $\mathrm{Y}$ motion and circular $\mathrm{X}$ and $\mathrm{Z}$ motion shown in Fig. 9(e), the projection of this helix in the plane normal to the Y motion is concentric. Conversely, for the other helix projections in Figs. 9(b) and (d), with linear motion in Z and X direction, respectively, significant off-axis components exist due to the discussed misalignments that cause the non-concentricity of these patterns. Furthermore, all projected patterns exhibit elliptic distortions and kinks that are likely to be caused by non-ideal motion and overshoots of the stage at the sinusoidal and co-sinusoidal turning points. These results clearly demonstrate that regardless of the alignment errors present, the proposed concept has the principle capability to fully characterise complex motion patterns and stage errors and that a future setup with improved alignment of the on-stage beamsplitters could be beneficial. 
(a)

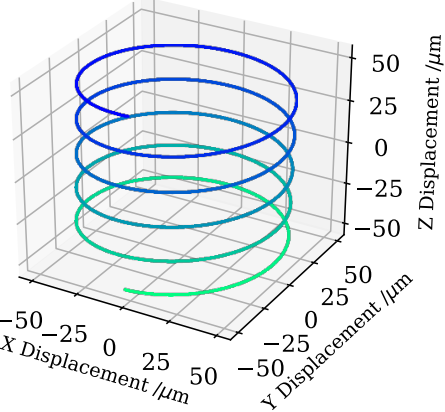

(c)

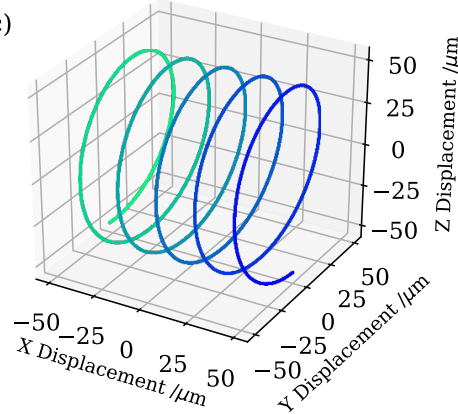

(e)

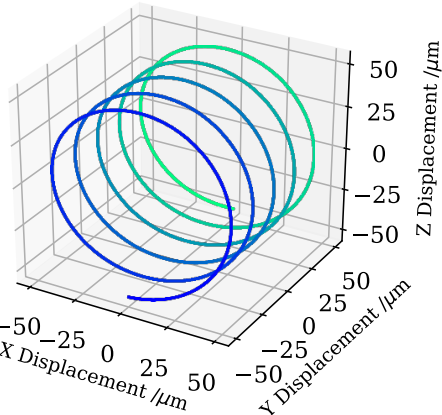

(b)

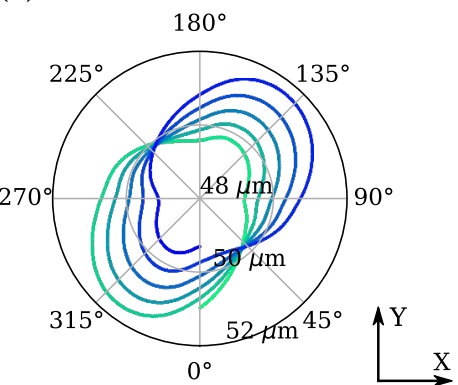

(d)

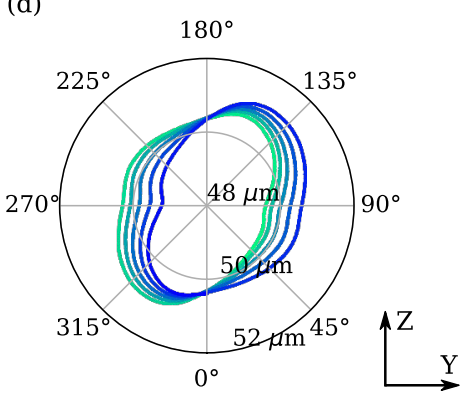

(f)

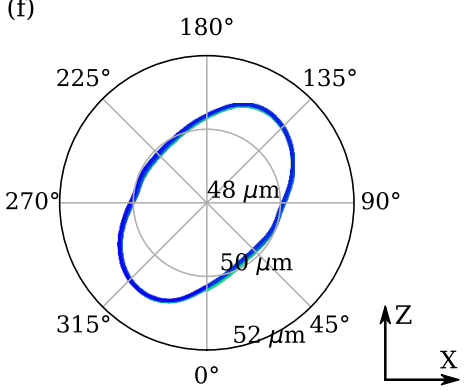

Figure 9: Graphs for combined helix stage motions, where the colour of the data has no physical significance and is used for the visualisation of the individual rotations. The measured displacements for a helical motion around the $\mathrm{Z}$ axis is shown in (a), while (b) shows a polar representation of these displacements projected into the X-Y plane. For helical motion around the $\mathrm{X}$ axis, (c) shows the measured displacements in all three dimensions, with (d) showing a polar representation projected into the Y-Z plane. The measured displacements for helical motion around the $\mathrm{Y}$ axis are shown in (e) and the polar representation projected into the $\mathrm{X}-\mathrm{Z}$ plane is shown in (f). 


\subsection{Further Discussion}

Whilst this experiment serves as a demonstration of the usefulness of this novel multi-dimensional stage encoder, it does not represent the maximum extent of the capabilities of this approach. The current system requires a minimum spatial separation between signal sources of approximately $5 \mathrm{~mm}$ (in air) to avoid cyclic errors due to peak crossovers. To use a more compact optical setup, the wavelength modulation amplitude could be increased through the use of more widely tunable diode lasers or, alternatively, through the use of external cavity lasers (ECL). However, particularly the use of ECLs is likely to increase the cost and lower the achievable quadrature bandwidth of the interferometer. With the current RRI interrogation hardware, using an ADC acquisition rate of $100 \mathrm{MHz}$ and operating at a wavelength modulation frequency of $24.4 \mathrm{kHz}$, required to achieve a quadrature bandwidth of $10 \mathrm{kHz}$, a maximum demodulation phase carrier amplitude of $\approx 2000 \mathrm{rad}$ can be interrogated[29]. This corresponds to a maximum distance (in air) from the fibre tip of $\approx 1.2 \mathrm{~m}$ using the previously determined scaling factor of $0.58 \mathrm{~mm} / \mathrm{rad}$. Lower wavelength modulation frequencies and correspondingly lower quadrature bandwidths could be chosen to increase the maximum distance, however, this would also lower the maximum travel speeds that are permissible. To determine the maximum allowable travel speed this setup can interrogate, we can use basic interferometric principles. The maximum phase change that can be measured over the inverse of the quadrature bandwidth $\mathrm{Q}$ is $2 \pi$, equivalent to one spatial period of the interrogation wavelength $\lambda$. The maximum travel speed that can be accurately measured is therefore $v=(\lambda / 2) \times Q$, where the factor of two arises as the setup operates in reflection. In this implementation, with $\mathrm{Q}=10 \mathrm{kHz}$ and $\lambda=1520.08 \mathrm{~nm}$, target velocities of up to $7.8 \mathrm{~mm} / \mathrm{s}$ can be tracked. The maximum travel range that can be measured using this approach is limited by the presence of undesired interferometers between the fibre tip and the beamsplitter surfaces. In this setup, as seen in Fig. 4(b), these form a grid with peaks at multiples of optical path of the beamsplitters of $15 \mathrm{~mm}$ (in air). If the desired interferometers $\alpha, \beta$ or $\gamma$ overlapped with this grid, increased cyclic errors would result. Therefore, 
with the current configuration, the maximum travel range is limited to $\pm 5 \mathrm{~mm}$, however, the maximum range of the proposed approach exceeds, by a factor of 100 , the $\pm 50 \mu \mathrm{m}$ maximum travel range for the Nanocube stage that was used here and which is representative of many nanopositioning systems with practical relevance.

This experiment demonstrates this approach for measurements in three dimensional Cartesian measurements, however variants and extensions of this setup could be envisioned that to allow the simultaneous measurement of other degrees of freedom, for example permitting angular measurements by evaluating the measurements from two spatially offset parallel beams for each mirror. In general, the setup could be simplified further through the removal of the $\mathrm{X}$ mirror and thus interferometer $\alpha$, and using only the interferometer $\delta$ between the reference surface $\mathrm{R}$ and the fibre tip to measure the $\mathrm{X}$ displacement. However, this would then make this measurement susceptible to vibrations in the beam delivery collimator fixture that may result in increased mechanical noise in the displacement signal. Whilst the dominant source of error in this proofof-principle experiment arises from misalignment due to the very crude method of aligning the beamsplitters using a 3D printed template, alignment errors could be easily reduced by a more sophisticated and rigorous on-stage mechanical setup that better aligns the beamsplitters to the stage motion axes. Other sources of errors include the absolute accuracy of the interrogation wavelength, where we attribute, using the manufacturer specifications for the described optical spectrum analyser measurements, an uncertainty of approximately 13 partsper-million (ppm). For the stage travel range of $\pm 50 \mu \mathrm{m}$ this corresponds to uncertainties of $\pm 0.7 \mathrm{~nm}$. For long-term measurements, laser wavelength stability must also be taken into account when considering sources of error, as it affects the entire effective path length of the interferometer. The laser driver and temperature controller used in this setup offer a combined stability of approximately $0.3 \mathrm{ppm}$ of the laser wavelength, which over the $99 \mathrm{~mm}$ effective path length of interferometer $\beta-\delta$, the longest effective path length used in this optical setup, adds a drift error of up to $30 \mathrm{~nm}$. In addition, in line with most 
interferometric techniques, air refractive index changes[39] will affect the uncertainty of measurements and also affect the entire path length and not just the travel range. Although a stable refractive index can be assumed for operation in well-controlled lab conditions, we expect approximately 1 ppm uncertainty, equivalent to a maximum error of up to $100 \mathrm{~nm}$, arising from this. Various techniques that are commonly applied in interferometry to reduce the uncertainty due to the air refractive index can be utilised here, such as the use of external refractometers[40] for compensation of the interferometric measurements. Furthermore, dual wavelength approaches[41] are also in principle compatible with the proposed approach. Future work on this system could incorporate the use of absolute wavelength calibration and stabilisation techniques such as absorption line-locking to gas cell, that would lower the absolute wavelength uncertainty and also improve the laser stability, which is necessary to achieve high-accuracy displacement measurements. Alternatively, a stable reference interferometer constructed using materials with low thermal expansion could easily be added to this setup and used to correct for both laser wavelength instability and refractive index changes[42].

\section{Conclusions}

In this paper, we have presented a novel approach to multi-dimensional positional encoders, using a range-resolved interferometric interrogation system and a simple, compact optical setup. We have demonstrated simultaneous, three-dimensional measurements for a variety of stage motions utilising a single optical access port, a single diode laser and a single photo detector, combining cost-effectiveness with a very high measurement performance. The presented measurements, over a nominal stage operating range of $\pm 50 \mu \mathrm{m}$ in all three Cartesian directions, have an instrumental noise density of below $0.4 \mathrm{~nm} / \sqrt{\mathrm{Hz}}$, with the noise floor dropping to below $0.1 \mathrm{~nm} / \sqrt{\mathrm{Hz}}$ above $50 \mathrm{~Hz}$, over a $10 \mathrm{kHz}$ quadrature bandwidth and achieve typical cyclic error amplitudes well below 1 $\mathrm{nm}$ without any corrections, a linearity performance comparable to many widely 
used but much more complex interferometer designs. In general, the approach presented in this work is very versatile and its compactness may open up novel possibilities for stage design. Additionally, the approach could also be extended to further interferometric configurations, for example incorporating angle measurements. The results obtained clearly demonstrate that this novel approach to multi-dimensional interferometric motion measurements has the potential to be widely used across many nanopositioning technology applications.

\section{Funding}

The authors acknowledge the support of the Engineering and Physical Sciences Research Council (EPSRC) UK via studentship EP/N509450/1 (Project: 1943924) and grants EP/N002520/1 and EP/M020401/1 as well as support from the Royal Society via Paul Instrument Fund grant PI150046 and the Royal Academy of Engineering via Research Fellowship RF/201718/1745.

\section{Acknowledgements}

The underlying data can be accessed at:

10.17862/cranfield.rd.11777853 from the Cranfield Online Research Data Repository (CORD).

\section{References}

[1] A. J. Fleming, A review of nanometer resolution position sensors: Operation and performance, Sensors and Actuators, A: Physical 190 (2013) 106-126. doi:10.1016/j.sna.2012.10.016.

[2] W. Gao, H. Haitjema, F. Z. Fang, R. K. Leach, C. F. Cheung, E. Savio, J. M. Linares, On-machine and in-process surface metrology for precision manufacturing, CIRP Annals - Manufacturing Technology 68 (2) (2019) 843-866. doi:10.1016/j.cirp.2019.05.005. 
[3] W. Gao, S. W. Kim, H. Bosse, H. Haitjema, Y. L. Chen, X. D. Lu, W. Knapp, A. Weckenmann, W. T. Estler, H. Kunzmann, Measurement technologies for precision positioning, CIRP Annals - Manufacturing Technology 64 (2) (2015) 773-796. doi:10.1016/j.cirp.2015.05.009.

[4] S. T. Smith, R. M. Seugling, Sensor and actuator considerations for precision, small machines, Precision Engineering 30 (3) (2006) 245-264. doi:10.1016/j.precisioneng.2005.10.003.

[5] Y. S. Choi, Y. Zhang, D. W. Lee, A thermal-driven silicon micro xystage integrated with piezoresistive sensors for nano-positioning, Journal of Micromechanics and Microengineering 22 (5) (2012) 055002. doi: $10.1088 / 0960-1317 / 22 / 5 / 055002$.

[6] R. K. Messenger, Q. T. Aten, T. W. McLain, L. L. Howell, Piezoresistive feedback control of a mems thermal actuator, Journal of Microelectromechanical Systems 18 (6) (2009) 1267-1278. doi:10.1109/JMEMS.2009. 2035370.

[7] M. Kim, W. Moon, E. Yoon, K. R. Lee, A new capacitive displacement sensor with high accuracy and long-range, Sensors and Actuators, A: Physical 130 (SPEC. ISS.) (2006) 135-141. doi:10.1016/j.sna.2005.12.012.

[8] H. Pu, H. Liu, X. Liu, K. Peng, Z. Yu, A novel capacitive absolute positioning sensor based on time grating with nanometer resolution, Mechanical Systems and Signal Processing 104 (2018) 705-715. doi:10.1016/j . ymssp. 2017.11.017.

[9] D. Zhang, S. Zhao, Q. Zheng, L. Lin, Absolute capacitive grating displacement measuring system with both high-precision and long-range, Sensors and Actuators, A: Physical 295 (2019) 11-22. doi:10.1016/j.sna. 2019. 05.027.

[10] V. G. Badami, P. J. de Groot, Displacement measuring interferometry, 
in: Handbook of Optical Dimensional Metrology, CRC Press, 2016, pp. 157-238. doi:10.1201/b13855-7.

[11] J. Rozman, A. Pleteršek, Linear optical encoder system with sinusoidal signal distortion below -60 dB, IEEE Transactions on Instrumentation and Measurement 59 (6) (2010) 1544-1549. doi:10.1109/TIM.2009.2027774.

[12] H. Haitjema, Achieving traceability and sub-nanometer uncertainty using interferometric techniques, Measurement Science and Technology 19 (8) (2008) 084002. doi:10.1088/0957-0233/19/8/084002.

[13] C. C. Wu, C. C. Hsu, J. Y. Lee, H. Y. Chen, C. L. Dai, Optical heterodyne laser encoder with sub-nanometer resolution, Measurement Science and Technology 19 (4) (2008) 045305. doi:10.1088/0957-0233/19/4/045305.

[14] C. B. Lee, S. K. Lee, Multi-degree-of-freedom motion error measurement in an ultraprecision machine using laser encoder - Review, Journal of Mechanical Science and Technology 27 (1) (2013) 141-152. doi: $10.1007 / \mathrm{s} 12206-012-1217-6$.

[15] P. J. de Groot, A review of selected topics in interferometric optical metrology, Reports on Progress in Physics 82 (5) (2019) 056101. doi: 10.1088/1361-6633/ab092d.

[16] S. Gibson, P. Coe, A. Mitra, D. Howell, R. Nickerson, Coordinate measurement in 2-D and 3-D geometries using frequency scanning interferometry, Optics and Lasers in Engineering 43 (7) (2005) 815-831. doi: 10.1016/j. optlaseng. 2004.09.001.

[17] N. Bobroff, Recent advances in displacement measuring interferometry, Measurement Science and Technology 4 (9) (1993) 907-926. doi:10.1088/ 0957-0233/4/9/001.

[18] C. Wang, L. long Xu, J. Zhu, Z. wen Yuan, Y. jie Yu, A. K. Asundi, A novel integrated fiber-optic interferometer model and its application in micro- 
displacement measurement, Optics and Lasers in Engineering 86 (2016) 125 - 131. doi:10.1016/j.optlaseng.2016.05.012.

[19] J. Guan, P. Köchert, C. Weichert, R. Köning, L. Siaudinyte, J. Flügge, A differential interferometric heterodyne encoder with 30 picometer periodic nonlinearity and sub-nanometer stability, Precision Engineering 50 (2017) 114-118. doi:10.1016/j.precisioneng. 2017.04.019.

[20] S. J. Cooper, C. J. Collins, A. C. Green, D. Hoyland, C. C. Speake, A. Freise, C. M. Mow-Lowry, A compact, large-range interferometer for precision measurement and inertial sensing, Classical and Quantum Gravity 35 (9) (2018) 095007. doi:10.1088/1361-6382/aab2e9.

[21] E. Stanfield, J. Stoup, M. Braine, T. Doiron, Sphere diameter interferometry with nanometer uncertainty, Metrologia 57 (2) (2020) 025003. doi:10.1088/1681-7575/ab5136.

[22] C.-C. Hsu, H. Chen, C.-W. Chiang, Y.-W. Chang, Dual displacement resolution encoder by integrating single holographic grating sensor and heterodyne interferometry, Optics Express 25 (24) (2017) 30189-30202. doi:10.1364/oe.25.030189.

[23] S. Agarwal, C. Shakher, In-plane displacement measurement by using circular grating talbot interferometer, Optics and Lasers in Engineering 75 (2015) 63 - 71. doi:10.1016/j.optlaseng. 2015.06.011.

[24] A. Kimura, W. Gao, Y. Arai, Z. Lijiang, Design and construction of a two-degree-of-freedom linear encoder for nanometric measurement of stage position and straightness, Precision Engineering 34 (1) (2010) 145-155. doi:10.1016/j.precisioneng.2009.05.008.

[25] C.-C. Wu, Y.-Z. Chen, C.-H. Liao, Common-path laser planar encoder, Optics Express 21 (16) (2013) 18872-18883. doi:10.1364/oe.21.018872.

[26] C. Lee, G. H. Kim, S. K. Lee, Design and construction of a single unit multifunction optical encoder for a six-degree-of-freedom motion error measure- 
ment in an ultraprecision linear stage, Measurement Science and Technology 22 (10) (2011) 105901. doi:10.1088/0957-0233/22/10/105901.

[27] S.-W. Pan, H.-L. Hsieh, W.-C. Wang, 6-DOF displacement and angle measurements using heterodyne laser encoder, in: Instrumentation, Metrology, and Standards for Nanomanufacturing, Optics, and Semiconductors VII, Vol. 8819, SPIE, 2013, pp. 38 - 45. doi:10.1117/12.2024082.

[28] Y. Shimizu, H. Matsukuma, W. Gao, Optical Sensors for Multi-Axis Angle and Displacement Measurement Using Grating Reflectors, Sensors 19 (23) (2019) 5289. doi:10.3390/s19235289.

[29] T. Kissinger, T. O. Charrett, R. P. Tatam, Range-resolved interferometric signal processing using sinusoidal optical frequency modulation, Optics Express 23 (7) (2015) 9415-9431. doi:10.1364/oe.23.009415.

[30] T. Kissinger, R. Correia, T. O. Charrett, S. W. James, R. P. Tatam, Fiber segment interferometry for dynamic strain measurements, Journal of Lightwave Technology 34 (19) (2016) 4620-4626. doi:10.1109/JLT. 2016.2530940 .

[31] T. Kissinger, E. Chehura, S. E. Staines, S. W. James, R. P. Tatam, Dynamic Fiber-Optic Shape Sensing Using Fiber Segment Interferometry, Journal of Lightwave Technology 36 (4) (2018) 917-925. doi:10.1109/ JLT . 2017.2750759.

[32] T. Kissinger, T. O. H. Charrett, S. W. James, A. Adams, A. Twin, R. P. Tatam, Simultaneous laser vibrometry on multiple surfaces with a single beam system using range-resolved interferometry, in: Optical Measurement Systems for Industrial Inspection IX, Vol. 9525, SPIE, 2015, pp. 470 - 476. doi:10.1117/12.2183281.

[33] K. B. Wiseman, T. Kissinger, R. P. Tatam, Two-dimensional remote interferometric stage encoder through a single access port using range-resolved 
interferometry, in: Optical Measurement Systems for Industrial Inspection XI, Vol. 11056, SPIE, 2019, pp. 63 - 69. doi:10.1117/12.2525596.

[34] P. L. M. Heydemann, Determination and correction of quadrature fringe measurement errors in interferometers, Applied Optics 20 (19) (1981) 33823384. doi:10.1364/A0.20.003382.

[35] T. G. McRae, M. T. L. Hsu, C. H. Freund, D. A. Shaddock, J. Herrmann, M. B. Gray, Linearization and minimization of cyclic error with heterodyne laser interferometry, Optics Letters 37 (13) (2012) 2448-2450. doi:10. 1364/o1.37.002448.

[36] B. Kelly, R. Phelan, D. Jones, C. Herbert, J. O'Carroll, M. Rensing, J. Wendelboe, C. Watts, A. Kaszubowska-Anandarajah, P. Perry, C. Guignard, L. Barry, J. O'Gorman, Discrete mode laser diodes with very narrow linewidth emission, Electronics Letters 43 (23) (2007) 1282-1284. doi:10.1049/el:20072311.

[37] A. Bergamin, G. Cavagnero, L. Cordiali, G. Mana, Beam-astigmatism in laser interferometry, IEEE Transactions on Instrumentation and Measurement 46 (2) (1997) 196-200. doi:10.1109/19.571811.

[38] Physik Instrumente Ltd UK, P-611.3 Datasheet, https://www. physikinstrumente.co.uk/fileadmin/user_upload/physik_ instrumente/files/datasheets/P-611.3-Datasheet.pdf (2019).

[39] B. Edlén, The refractive index of air, Metrologia 2 (2) (1966) 71-80. doi : $10.1088 / 0026-1394 / 2 / 2 / 002$.

[40] M. L. Eickhoff, J. L. Hall, Real-time precision refractometry: new approaches, Applied Optics 36 (6) (1997) 1223-1234. doi:10.1364/ao.36. 001223.

[41] Z. Liu, W. Li, Bayanheshig, X. Li, S. Jiang, Y. Song, Q. Lv, Two-color heterodyne laser interferometry for long-distance stage measurement with 
correction of uncertainties in measured optical distances, Scientific Reports 7 (1) (2017) 1-10. doi:10.1038/s41598-017-07741-4.

[42] O. Gerberding, K. S. Isleif, M. Mehmet, K. Danzmann, G. Heinzel, LaserFrequency Stabilization via a Quasimonolithic Mach-Zehnder Interferometer with Arms of Unequal Length and Balanced dc Readout, Physical Review Applied 7 (2017) 024027. doi:10.1103/PhysRevApplied.7.024027. 
2020-08-30

\section{Three-dimensional interferometric stage encoder using a single access port}

Wiseman, Kieran B.

Elsevier

Wiseman KB, Kissinger T, Tatam RP. (2021) Three-dimensional interferometric stage encoder using a single access port. Optics and Lasers in Engineering, Volume 137, February 2021 , Article number 106342

https://doi.org/10.1016/j.optlaseng.2020.106342

Downloaded from Cranfield Library Services E-Repository 